\title{
The volatile inventory and hydrogen isotope composition of apatite in the Brachina meteorite
}

\author{
ROMAIN TARTESE ${ }^{1}$, YVES MARROCCHI ${ }^{2}$, LAURETTE \\ PIANI $^{3}$ AND MAHESH ANAND ${ }^{4}$ \\ ${ }^{1}$ The University of Manchester \\ ${ }^{2} \mathrm{CRPG}$, CNRS-Université Lorraine \\ ${ }^{3} \mathrm{CRPG}$ \\ ${ }^{4}$ The Open University \\ Presenting Author: romain.tartese@manchester.ac.uk
}

Water is essential for life as we know it. Therefore, understanding the origin(s) and transport of water across the Solar System is of primary importance, and is an overarching science question driving the Solar System exploration. Models investigating this issue generally rely on the records preserved in meteorites sourced from the asteroid belt, and notably in the volatile-rich carbonaceous chondrite meteorites, because they are thought to 'best' represent the starting composition of the protosolar nebula [1]. However, the parent bodies of these samples accreted relatively late compared with some other asteroidal bodies [2]. Thus, it is important to investigate alternative records accessible in 'older' meteorite reservoirs.

Partially-melted asteroids, sampled by the achondrite meteorites, provide us with such a complementary archive. The asteroidal parent bodies of achondrites such as the angrites and the ureilites accreted very early, within $c a$. 1 million years (Myr) after the start of Solar System formation (compared to $c a .+3-4$ Myr for carbonaceous chondrites parent bodies [2]). Recent secondary ion mass spectrometry (SIMS) studies have established the water inventory and $\mathrm{H}$ isotope composition of meteorite samples from the howardite-eucrite-diogenite and the angrite parent bodies [3-5], which appear to share a common origin with water in volatile-rich carbonaceous chondrites. To complement this limited achondrite dataset, we used SIMS to investigate the $\mathrm{F}-\mathrm{Cl}-\mathrm{OH}$ abundance and $\mathrm{H}$ isotope composition of apatite in Brachina, whose parent-body partially melted within ca. 1 Myr after the start of Solar System formation [6]. Our results show that Brachina apatite is $\mathrm{Cl}$-rich, containing $~ 5.8$ wt. $\% \mathrm{Cl}$ and $\sim 0.6$ wt.\% F. Apatite $\mathrm{H}_{2} \mathrm{O}$ abundances range between $\sim 40$ and $3000 \mu \mathrm{g} . \mathrm{g}^{-1}$, and are negatively correlated with $\delta \mathrm{D}$ values decreasing from $\sim+3500$ to $-150 \%$. We will discuss these results further at the conference.

References: [1] Robert (2006) Meteorites and the Early Solar System II, Univ. Arizona Press, p. 341. [2] Sugiura \& Fujiya (2014) MaPS 49, p. 772. [3] Sarafian et al. (2014) Science 346, p. 623. [4] Barrett et al. (2016) MaPS 51, p. 1110. [5] Sarafian et al. (2017) PTRS A 375, p. 20160209. [6] Dunlap et al. (2017) LPSC XLVIII, Abstract \#2981. 